\title{
Recalcitrant steroid-resistant EGPA with multisystem involvement
}

\author{
Andrea Cervi ${ }^{1}$, Gerard Cox $^{2}$, Nader Khalidi ${ }^{3}$, Parameswaran Nair ${ }^{2}$, Nathan Hambly ${ }^{* 2}$ \\ ${ }^{1}$ Internal Medicine Residency Program, McMaster University, Hamilton, Canada \\ ${ }^{2}$ Division of Respirology, McMaster University, Hamilton, Canada \\ ${ }^{3}$ Division of Rheumatology, McMaster University, Hamilton, Canada
}

Received: March 3, 2017

DOI: $10.5430 /$ crim.v4n $3 p 12$
Accepted: May 22, 2017

Online Published: June 6, 2017

\begin{abstract}
Eosinophilic granulomatosis with polyangiitis (EGPA) is a multisystem disorder classically characterized by sequential histopathologic and clinical disease phases. An eosinophilic tissue infiltration phase typically precedes the development of a systemic small-vessel vasculitic phase. The contribution of these two pathogenic mechanisms to disease course and the boundaries between them are often blurred. We report the case of a 49-year-old man with established p-ANCA positive EGPA presenting acutely with central and peripheral nervous system dysfunction, diffuse alveolar hemorrhage, biopsy-proven myositis, and myocarditis resistant to steroids. The patient's clinical course was marked by unremitting abdominal pain associated with significant weight loss. Although eosinophilic infiltrates were not observed on gastrointestinal mucosal biopsy, samples stained heavily for eosinophil peroxidase, indicating prior degranulation of eosinophils. Complete remission of eosinophil-mediated tissue infiltration and vasculitic phases was achieved only after the addition of intravenous cyclophosphamide to corticosteroid therapy. This case report demonstrates both: a) the importance of recognizing eosinophilic degranulation in addition to intact eosinophils in tissue and b) a unique presentation of a rare disease, underscoring the multifaceted and heterogeneous nature of EGPA and its overlapping disease phases.
\end{abstract}

Key Words: Anti-neutrophilic cytoplasmic antibodies, Eosinophilic granulomatosis with polyangiitis, Eosinophil peroxidase, Gastrointestinal tract

\section{INTRODUCTION}

Eosinophilic granulomatosis with polyangiitis (EGPA) is a rare systemic disease whose clinical manifestations arise from two pathogenic processes: eosinophilic tissue infiltration and necrotizing vasculitis. Traditionally, the course of EGPA has been described as evolving through prodromal, eosinophil-mediated and vasculitic phases. ${ }^{[1,2]}$ Specifically, organ damage secondary to eosinophil infiltration underlies airway and lung parenchymal, cardiac and gut involvement, while mononeuritis multiplex, glomerulonephritis and purpura characterize the vasculitic phase. ${ }^{[3-5]}$

We report the clinical disease course of a gentleman with steroid-resistant EGPA characterized by recalcitrant peripheral eosinophilia, central and peripheral nervous system involvement, myocarditis, diffuse alveolar hemorrhage (DAH), necrotizing myopathy, and extensive eosinophilic degranulation in gastrointestinal tissues; the latter only apparent after staining with targeted monoclonal antibodies to eosinophil

\footnotetext{
*Correspondence: Nathan Hambly, MD, FRCPC; Email: nathan.hambly@medportal.ca; Address: Firestone Institute for Respiratory Health, St. Joseph's Healthcare Hamilton, 50 Charlton Avenue East, Hamilton, ON, L8N4A6, Canada.
} 
peroxidase (EPX). This case highlights the synchronous presentation of multiple rare manifestations of EGPA, highlighting the multisystem complexity and clinical heterogeneity of this disease entity.

\section{CASE PRESentation}

A 49-year-old man was admitted to hospital for acute onset vertigo and progressive bilateral leg pain. The patient's medical history included EGPA manifested by asthma, recurrent sinusitis, transient pulmonary infiltrates, peripheral eosinophilia, and positive p-ANCA serology. Prior to his acute deterioration, his disease had been successfully man- aged with azathioprine (150 $\mathrm{mg}$ daily), prednisone (5 mg daily) and inhaled corticosteroids.

The patient's vital signs on admission included a heart rate of 80 beats per minute, blood pressure $145 / 87 \mathrm{mmHg}$, respiratory rate 18 breaths per minutes, oxygen saturation $97 \%$ on room air, and temperature of $36.8^{\circ} \mathrm{C}$. Respiratory and cardiovascular system examinations were normal. Abdomen was soft and not distended and was not tender to light or deep palpation. There was no palpable organomegaly. Both upper and lower limb girdle muscle strengths were preserved. Deep tendon reflexes were normal as were tests of cerebellar function.

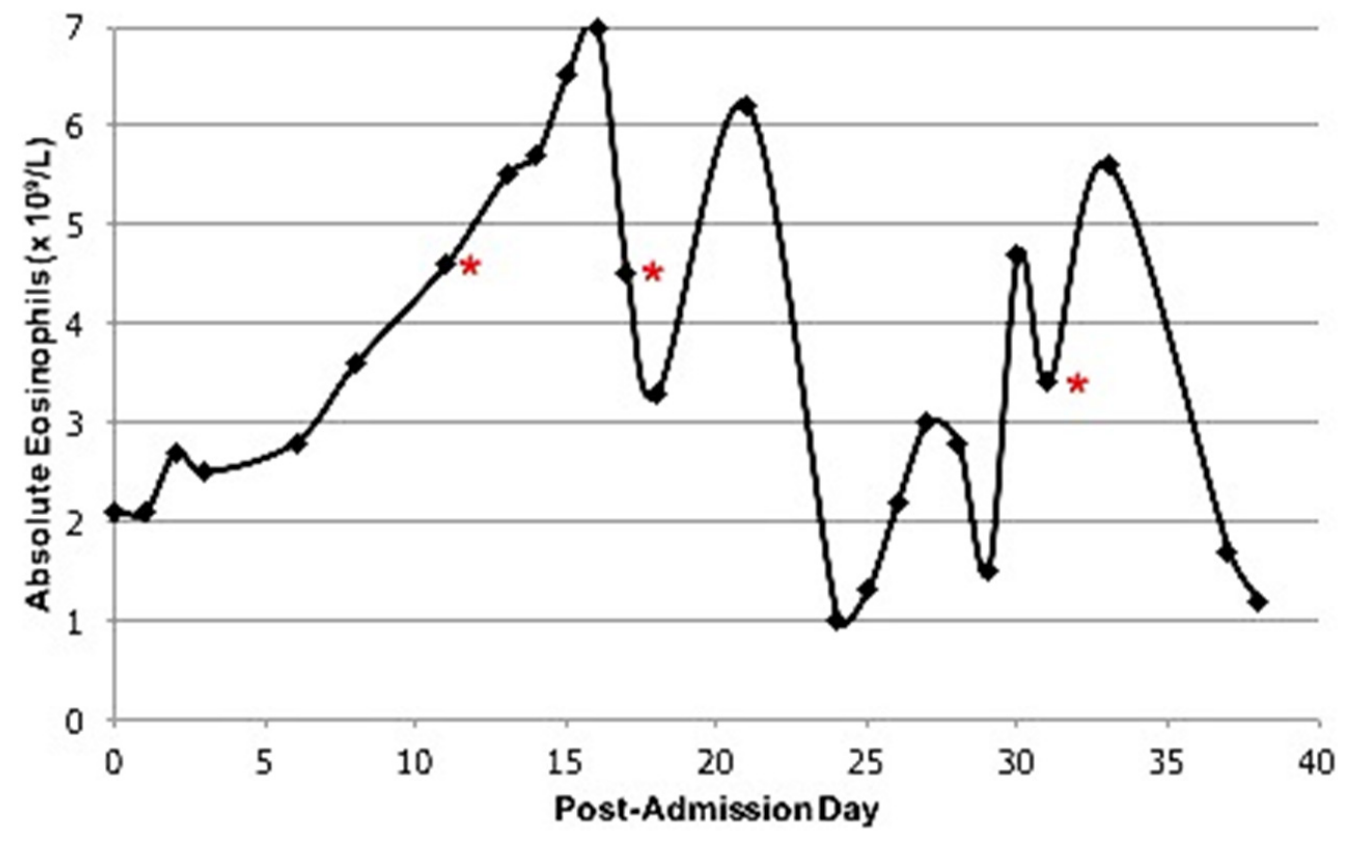

Figure 1. Graphical trend of peripheral eosinophilia throughout patient's admission. Red symbols indicate escalation of immunosuppression, initially in the form of increased prednisone dosage ( $20 \mathrm{mg}$ to $60 \mathrm{mg}$ once daily) on post-admission day 11 , then to IV methylprednisolone (40 mg daily) on post-admission day 17 . Pulse methylprednisolone (1,000 mg) and cyclophosphamide infusion were initiated on post-admission day 31.

Admission bloodwork revealed an elevated eosinophil count of $2.1 \times 10^{9} / \mathrm{L}$. (Figure 1 demonstrates a graphical trend of the patient's peripheral eosinophil count throughout his admission.) The patient's basic metabolic profile, serum creatinine, and aminotransferases were within normal limits. An asymptomatic elevation of high-sensitivity troponin was observed $(0.70 \mu \mathrm{g} / \mathrm{L})$. Subsequent cardiac MRI noted patchy delayed gadolinium enhancement, consistent with myocarditis. MRI of his head to evaluate vertigo revealed multiple acute right cerebellar infarcts. Biochemical evidence of myositis was noted with elevated CK (630 U/L). MRI of the pelvis showed myositis involving the gluteus maximus muscles. Muscle biopsy demonstrated focal necro- tizing myopathy with moderate microangiopathy, related to vasculitis.

In response to his persistent vertigo, new myocarditis, myopathy, and prominent peripheral eosinophilia, prednisone was increased to $60 \mathrm{mg}$ daily. Despite this measure, the patient developed disabling mononeuritis multiplex involving his left ulnar and median nerves, which was confirmed by electromyography/nerve conduction study. Bone marrow examination for clonal proliferation, molecular cytogenetics and flow cytometric analysis for abnormal T-cell population and PCR for T-cell receptor rearrangements excluded a malignant hematologic process or hypereosinophilic syndrome as alternative explanations for the patient's deteriorating clin- 
ical course and increasing peripheral eosinophilia. While treated with high-dose corticosteroids the patient developed severe, diffuse abdominal pain that was associated with a 50-lb. weight loss.

Esophagogastroduodenoscopy and flexible sigmoidoscopy were normal and did not provide an explanation for the patient's severe abdominal pain. There was no evidence of eosinophil infiltration or features suggestive of vasculitis on histologic evaluation. However, immunohistochemistry staining using a targeted monoclonal antibody against EPX, ${ }^{[6]}$ an eosinophil derived granule protein, revealed extensive activity in samples from the esophagus, duodenum and stomach indicating prior eosinophil infiltration, activation and subsequent degranulation (see Figure 2).
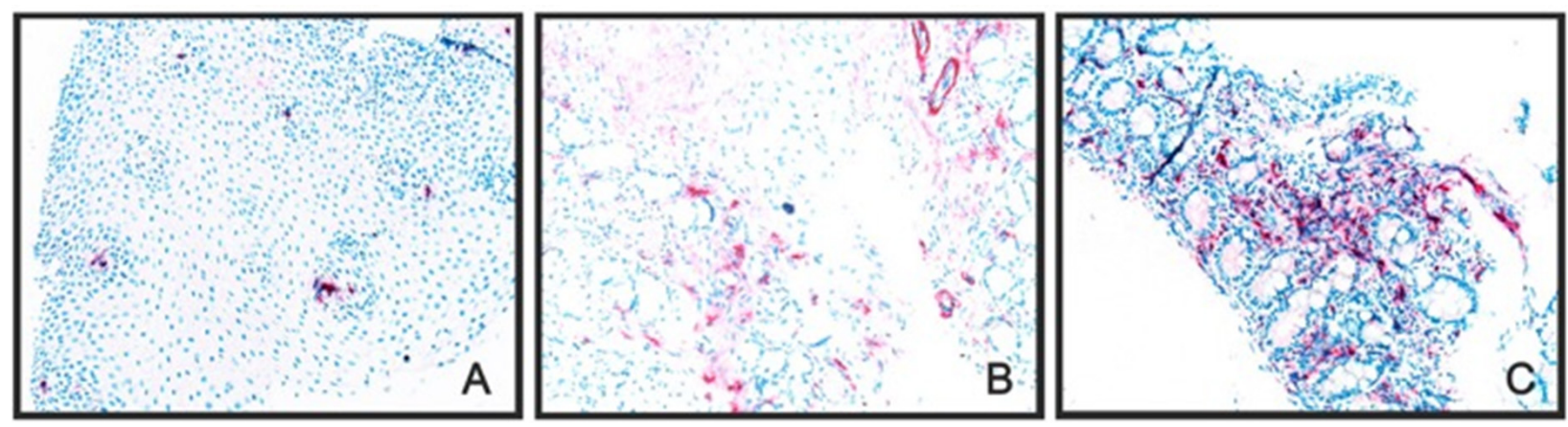

Figure 2. Gastrointestinal biopsy specimens demonstrating eosinophil peroxidase immunoreactivity in the A) esophagus $(\times 20)$, B) stomach $(\times 20)$ and $C)$ duodenum $(\times 20)$, indicating degranulation of eosinophils in the tissues.

Prior to further escalation of immunosuppression, the patient underwent bronchoscopy as he had been treated for Aspergillus fumigatus tracheobronchitis two months prior. CT scan of the chest completed during his hospital course identified multiple ill-defined ground-glass nodules and airspace densities (see Figure 3). Methicillin-sensitive Staphylococcus aureus and Aspergillus ustus were isolated from the bronchoalveolar lavage (BAL) fluid. The patient was treated for two weeks with cefazolin and voriconazole. Given the plan to escalate his immunosuppression, a repeat BAL was performed to confirm microbial eradication. Although the patient remained asymptomatic from a respiratory perspective, serial washings revealed diffuse alveolar hemorrhage, while all cultures were negative.

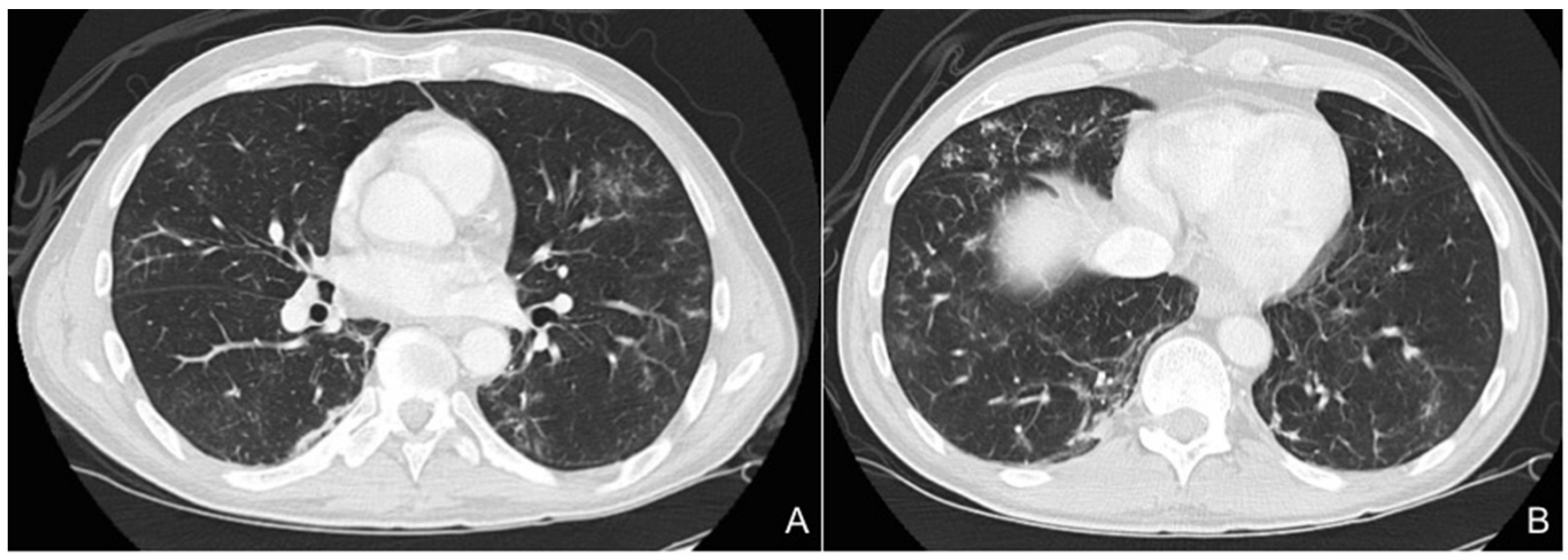

Figure 3. Representative images of CT chest showing multiple ill-defined ground glass nodules with lower lobe predominance and small areas of airspace disease. Fluid from bronchoalveolar lavage grew methicillin-sensitive Staphylococcus aureus and Aspergillus ustus, for which the patient was treated. Repeat bronchoscopy post-antibiotics demonstrated evidence of diffuse alveolar hemorrhage.

Based on deteriorating neuromuscular disease, DAH and demonstration of an intense eosinophilic gastroenteritis, the patient was treated with IV cyclophosphamide $(15 \mathrm{mg} / \mathrm{kg})$ and IV methylprednisolone (1,000 mg daily for three days).
The patient's previously intractable symptoms improved dramatically. The patient's only residual symptom at the time of discharge was persistent left-hand weakness, secondary to mononeuritis, which improved with long-term physiotherapy. 
The patient was discharged home on oral prednisone (50 $\mathrm{mg} /$ day) and received a total of 10 doses of IV cyclophosphamide as an outpatient. Currently, he remains in remission and is managed with oral methotrexate (20 mg weekly) and prednisone (10 $\mathrm{mg}$ daily), along with high dose inhaled fluticasone (1,000 $\mu \mathrm{g}$ twice daily) and inhaled salmeterol (50 $\mu \mathrm{g}$ twice daily). The patient's most recent blood eosinophil count was $0.5 \times 10^{9} / \mathrm{L}$.

\section{Discussion}

This case describes the synchronous presentation of multiple rare (ischemic cerebellar infarcts, necrotizing myositis, DAH, and GI eosinophilic infiltration) and common (myocarditis and mononeuritis multiplex) manifestations of EGPA. In a large systematic retrospective study, only $2.1 \%$ of patients with EGPA developed ischemic stroke during the 5-year follow-up period; ${ }^{[7]} \mathrm{DAH}$ was present in only $4.2 \%$ of patients. The concurrent presentation of these two symptoms alone highlights the rarity of our patient's initial presentation. ${ }^{[8,9]}$ The presence of disabling myalgia at the time of hospitalization was also notable, as myalgia rarely represents a main presenting feature of EGPA and is most commonly seen during the prodromal phase of the illness. ${ }^{[4]}$

Gastrointestinal involvement in EGPA is thought to be eosinophil-mediated, ${ }^{[10,11]}$ which was not readily apparent on initial histopathologic evaluation of our patient's intestinal biopsy specimens. Staining with a monoclonal antibody targeting EPX however revealed intense staining in biopsy specimens taken from multiple sites in the gastrointestinal tract, providing evidence of prior eosinophil degranulation and pathophysiologic insight into our patient's unremitting abdominal pain. EPX is a secondary granule protein uniquely secreted by eosinophils. EPX is easily detected and quantified using a novel monoclonal antibody and strongly correlates with eosinophil-mediated inflammation in nasal, sputum and BAL samples. ${ }^{[6,12]}$ This case demonstrates the critical utility of this assay as a valuable complement to conventional methods that identify intact eosinophils, in the evaluation of suspected eosinophil-mediated tissue inflam- mation.

Disease remission, with near-complete resolution of symptoms, was rapidly achieved following initiation of IV cyclophosphamide. As cyclophosphamide is traditionally utilized to target vasculitis rather than eosinophil-mediated inflammation it was somewhat surprising to observe such a rapid global therapeutic response. This highlights the lack of clearly-defined pathophysiologic roles for eosinophils and blood vessel inflammation in EGPA pathogenesis and disease course. ${ }^{[13]}$ Mepolizumab, a monoclonal antibody targeting interleukin-5 (IL-5), was recently studied in an EGPA population with relapsing or refractory EGPA. ${ }^{[14]}$ As IL-5 is a key mediator of eosinophil production and activation, it is postulated that anti-IL-5 therapy would target the eosinophil component of EGPA. Although treatment with mepolizumab resulted in significantly more weeks in remission and a reduction in glucocorticoid dose, only approximately $50 \%$ of patients treated with active therapy had protocol defined remission. Since the majority of patients who entered this clinical trial did not have active vasculitis or a vasculitisrelated "flare" within a six-month period of participating in the study, it is not clear if targeting the eosinophilic inflammation directly with an anti-eosinophil drug would be effective to prevent a recurrence of vasculitis.

In summary, we report a rare concurrence of multisystem involvement in EGPA, highlighting the utility of targeted diagnostic testing in the setting of a complex clinical presentation and that our understanding of the factors contributing to the clinical expression and natural history of EGPA continue to be poorly understood.

\section{ACKNOWLEDgements}

We are extremely grateful to Dr. Jamie Lee and Dr. Sergei Ochkur, Mayo Clinic, Scottsdale, for their help with the EPX immunohistochemistry.

\section{CONFLicts OF INTEREST Disclosure}

The authors have declared no conflicts of interest.

\section{REFERENCES}

[1] Mouthon L, Dunogue B, Guillevin L. Diagnosis and classification of eosinophilic granulomatosis with polyangiitis (formerly named Churg-Strauss syndrome). Journal of Autoimmunity. 2014; 55 (4849): 99-103. PMid:24530234 https://doi.org/10.1016/j.ja ut. 2014.01 .018

[2] Greco A, Rizzo MI, De Virgilio A, et al. Churg-Strauss syndrome. Autoimmunity Reviews. 2015; 14(4): 341-8. PMid:25500434 https : //doi.org/10.1016/j.autrev.2014.12.004

[3] Mahr A, Moosig F, Neumann T, et al. Eosinophilic granulomatosis with polyangiitis (Churg-Strauss): Evolutions in classification, etiopathogenesis, assessment and management. Current Opinions in Rheumatology. 2014; 26(1): 16-23. PMid:24257370 https: //doi.org/10.1097/BOR.0000000000000015

[4] Baldini C, Talarico R, Della Rossa A, et al. Clinical manifestations and treatment of Churg-Strauss syndrome. Rheumatic Disease Clinics of North America. 2010; 36(3): 527-43. PMid:20688248 https://doi.org/10.1016/j.rdc.2010.05.003

[5] Khoury P, Grayson PC, Klion AD. Eosinophils in vasculitis: Characteristics and roles in pathogenesis. Nat Rev Rheumatol. 2014; 10(8): 474-83. PMid:25003763 https://doi.org/10.1038/nrrheum. 2014.98 
[6] Nair P, Ochkur SI, Protheroe C, et al. Eosinophil peroxidase in sputum represents a unique biomarker of airway eosinophilia. Allergy. 2013; 68(9): 1177-84. https://doi.org/10.1111/all.12206

[7] Comarmond C, Pagnoux C, Khellaf M, et al. Eosinophilic granulomatosis with polyangiitis (Churg-Strauss): clinical characteristics and long-term follow-up of the 383 patients enrolled in the French Vasculitis Study Group cohort. Arthritis Rheum. 2013 65(1): 270-81. PMid:23044708 https ://doi.org/10.1002/art. 37721

[8] West S, Arulkumaran N, Ind PW, et al. Diffuse alveolar hemorrhage in ANCA-associated vasculitis. Intern Med. 2013; 52: 5-13. PMid:23291668 https://doi.org/10.2169/internalmedici ne. 52.8863

[9] Kostianovsky A, Hauser T, Pagnoux C, et al. Alveolar hemorrhage in ANCA-associated vasculitides: 80 patients' features and prognostic factors. Clin Exp Rheumatol. 2012; 30 (1): S77-82. PMid:22640651

[10] Assmann G, Molinger M, Pfreundschuh M, et al. Gastrointestinal perforation due to vasculitis at primary diagnosis of eosinophilic granulomatosis with polyangiitis (EGPA) despite a high dose glucocor- ticosteroids treatment. SpringerPlus. 2014; 3: 404. PMid:25133090 https://doi.org/10.1186/2193-1801-3-404

[11] Franco DL, Ruff K, Mertz L, et al. Eosinophilic granulomatosis with polyangiitis and diffuse gastrointestinal involvement. Case Reports Gastroenterol. 2014; 8: 329-36. PMid:25473392 https: //doi.org/10.1159/000369129

[12] Rank MA, Ochkur SI, Lewis JC, et al. Nasal and pharyngeal eosinophil peroxidase levels in adults with poorly controlled asthma correlate with sputum eosinophilia. Allergy. 2016; 71(4): 567-70. PMid:26645423 https://doi.org/10.1111/all.12817

[13] Chaigne B, Terrier B, Theilblemont N, et al. Dividing the Janus vasculitis? Pathophysiology of eosinophilic granulomatosis with polyangiitis. Autoimmun Rev. 2016; 15(2): 139-45. PMid:26506114 https://doi .org/10.1016/j. autrev. 2015.10.006

[14] Wechsler ME, Akuthota P, Jayne D, et al. Mepolizumab or placebo for eosinophilic granulomatosis with polyangiitis. N Engl J Med. 2017; 376: 1921-32. PMid:28514601 https://doi.org/10.105 6/NEJMoa1702079 